\title{
Silicon carbide detectors study for NUMEN project
}

\begin{abstract}
A. Muoio ${ }^{1,2}$, C. Agodi $^{1}$, D.L. Bonanno ${ }^{3}$, D.G. Bongiovanni ${ }^{3}$,
\end{abstract} S. Calabrese ${ }^{1.4}$, L. Calcagno ${ }^{3.4}$, D. Carbone $^{1}$, M. Cavallaro $^{1}$,

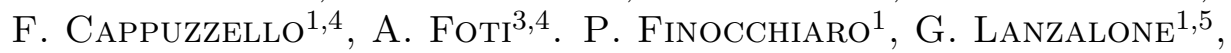
F. La Via ${ }^{6}$, F. Longhitano ${ }^{3}$, D. Lo Prestit ${ }^{3,4}$, L. PANdola ${ }^{1}$, S. Privitera ${ }^{6}$ and S. Tudisco ${ }^{1}$

\author{
${ }^{1}$ INFN - Laboratori Nazionali del Sud, Catania, Italy \\ ${ }^{2}$ Dip. di Fisica e Scienze della Terra, Univ. di Messina, Messina, Italy \\ ${ }^{3}$ INFN, Sezione di Catania, Catania, Italy \\ ${ }^{4}$ Dip. di Fisica e Astronomia, Univ. di Catania, Catania, Italy \\ ${ }^{5}$ Univ. degli Studi di Enna Kore, Enna, Italy \\ ${ }^{6}$ CNR-IMM, Catania, Italy
}

\begin{abstract}
In this contribution, we will illustrate the main results of the R\&D activities related to the Silicon Carbide detectors associated with NUMEN project.
\end{abstract}

\section{NUMEN Physics}

The NUMEN project[1-4] proposes an innovative technique to access the nuclear matrix elements entering the expression of the half-life of the double beta decay by relevant cross sections measurements of heavy-ion induced double charge exchange (DCE) reactions, $\left({ }^{18} \mathrm{O},{ }^{18} \mathrm{Ne}\right),\left({ }^{20} \mathrm{Ne},{ }^{20} \mathrm{O}\right)$ and $\left({ }^{12} \mathrm{C},{ }^{12} \mathrm{Be}\right)$. DCE reactions will be investigated at the INFN-LNS laboratory with incident energies ranging from 10 to $60 \mathrm{MeV} / \mathrm{A}$. A key aspect of the project is the use of the MAGNEX [5-6] large acceptance magnetic spectrometer to detect the ejectiles produced in the nuclear collisions[7]. With 
the present set-up it is difficult to suitably extend this research to the hot cases, where $\beta \beta$ decay studies are and will be concentrated. The present limitations concern mainly the low beam current from the L.N.S. superconducting cyclotron CS accelerator and the low counting rate tolerated $(<5$ $\mathrm{kHz}$ )[8-9] by the MAGNEX Focal Plane Detector (FPD). In the view of a systematic study of the many hot cases of $\beta \beta$ decays, an upgraded setup, able to work with at least two orders of magnitude more current than the present, is thus mandatory. This goal can be achieved by a substantial change in the technologies used in the beam extraction and transport and in the detection of the ejectiles. For the spectrometer, the main foreseen upgrade is the substitution of the present FPD. In particular the existing wall of stopping detectors based on $50 \times 70 \mathrm{~mm}^{2}, 0.5 \mathrm{~mm}$ thick silicon pad detectors needs to be upgraded in view of the higher detection rate. Many requirements must be taken into account for the design of the new stopping wall telescopes for particle identification. The most relevant aspects are: a) energy resolution better than $1 \%$ to maintain the performances in the particle identification reached with the present set-up of the FPD (mass resolution 1/160) [10]; b) unambiguous and fast particle identification procedure; c) time resolution better than $1 \div 2 \mathrm{~ns}$ in order to guarantee an accurate measurement of the drift time in the gas chamber used to reconstruct the vertical track of the ejectiles; d) a high granularity(modules of $1 \mathrm{~cm}^{2}$ ) is required in order to limit double-hit events probability below $10 \%$ in the whole FPD; e) the thickness of the telescope must be chosen in order to permit the detection of the ejectiles in the wide dynamical range of incident energies (from 10 up to $60 \mathrm{MeV} / \mathrm{A}$ ). In this context, the choice of solid state detectors, such as $\mathrm{SiC}$, is the most appropriate.

\section{Silicon carbide}

Radiation damage can affect various properties of a detector. Phenomena connected with the alteration of these properties are the increase of the leakage current, the decrease of charge collection efficiency (CCE) and the removal of free carriers from the conductive regions of the device. Radiation hardness is the inertness of these device parameters to high doses of particle irradiation [11]. SiC, due to its wide gap and strength of its chemical bonds, has been seriously considered as a valid alternative to Si for the production of radiation hard ionizing particle detectors. The leakage current of a p-n junction consists of diffusion current from the quasi-neutral areas and generation current from depletion area [12]. The second term depends 
essentially on the temperature $\mathrm{T}$ and on the energy gap $\mathrm{E}_{g}$. Increasing the $\mathrm{E}_{g}$ from $1.1 \mathrm{eV}(\mathrm{Si})$ to $3.2 \mathrm{eV}(4 \mathrm{H}-\mathrm{SiC})$ determines a reduction of the diffusion term of the leakage current of about 17 orders of magnitude at room temperature $(\sim 300 K)$. Silicon Carbide technology offers then an ideal response to such challenges, since it gives the opportunity to cope the excellent properties of silicon detectors (resolution, efficiency, linearity compactness) with a much bigger radiation hardness (up to five orders of magnitude for heavy ions), thermal stability and insensitivity to visible light. R\&D activity is mandatory to develop innovative processes, which allows a massive production of thick $(>100 \mu \mathrm{m})$ and large area (about $1 \mathrm{~cm}^{2}$ ) SiC detectors. Following these indications we started the research work with a Monte Carlo simulation with the aim to study $\Delta \mathrm{E}$ - E configuration (with respective thicknesses $100 \mu \mathrm{m}$ and $1000 \mu \mathrm{m}$ ) from the point of view of defects dislocation generated by $\mathrm{an}^{18} \mathrm{O}$ at $25 \mathrm{MeV} / \mathrm{A}$. For this work we used the SRIM [13] software; it is a collection of packages which calculate many features of the transport of ions in matter. The following figure 1.a shows the number of simulated vacancies as function of protons (at $25 \mathrm{MeV}$ and $1 \mathrm{GeV}$ ) and ${ }^{18} \mathrm{O}$ $(25 \mathrm{MeV} / \mathrm{A})$ ions fluency. One can notice that the number defects create in the same stage $(\Delta \mathrm{E}$ or $\mathrm{E})$ from protons beam at $25 \mathrm{MeV} / \mathrm{A}$ is about two orders of magnitude smaller then the corresponding number coming from heavy oxygen ions of $25 \mathrm{MeV} / \mathrm{A}$. In Figure1.b, we show the results of such calculation, we can be notice as several order of magnitude of leakage current increased are expected at high doses irradiation/fluency. Such increase could be acceptable for $\mathrm{SiC}$ detectors, they have a leakage current five order of magnitude less than Silicon detectors. (Where the maximum of acceptable fluency is about $10^{9}$ heavy ions $/ \mathrm{cm}^{3}$ ).

We have studied, moreover, the effects of the irradiation comparing $\mathrm{SiC}$ and $\mathrm{Si}$ detectors. These detectors were irradiated with $\mathrm{C}^{+}$ions at $740 \mathrm{MeV}$ and at different doses. For the experiment we used a standard Si detector of $1 \mathrm{~cm}^{2}$ of active area and $300 \mu \mathrm{m}$ thickness and $\mathrm{SiC}$ detector which was built growing a $4 \mathrm{H}-\mathrm{SiC}$ n-type epitaxial layers with a carrier concentration of $1.0 \cdot 10^{-14} \mathrm{~cm}^{-3}$ and a thickness of $80 \mu \mathrm{m}$ on a highly doped n-type $\left(\sim 7.0 \cdot 10^{-18} \mathrm{~cm}^{-3}\right)$ substrate. The following Table 1 shows the results of the electrical static characterization before and after the irradiation. Can be observe as Si detectors are acceptable to work at the irradiation 94kGy dose; while $\mathrm{SiC}$ are able to survive at $218 \mathrm{kGy}$ dose. 


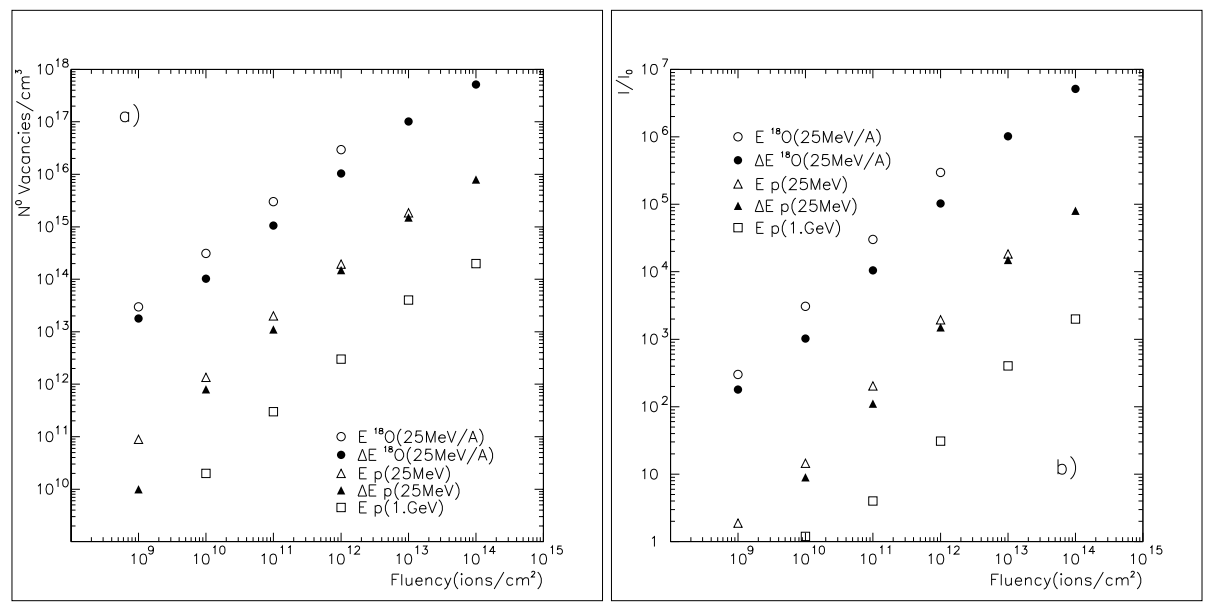

Figure 1: The figure 1.a shows the comparison of Number of silicon vacancies\%Fluency on $\Delta \mathrm{E}$ and $\mathrm{E}$ in $\mathrm{SiC}$ stages; while the figure 1.b shows the comparison of Increment of current leakage\%Fluency in $\mathrm{SiC}$ for thickness of $\Delta \mathrm{E}$ and E. In both figures the solid symbols indicate the first stage of the detector (with a thickness of $100 \mu \mathrm{m}$ ), while the empty symbols the second stage (with a thickness of $1000 \mu \mathrm{m})$. The following energies has been simulated: oxygen ions at $25 \mathrm{MeV} / \mathrm{A}$ (circle), protons ions at $25 \mathrm{MeV} / \mathrm{A}$ (triangle) and protons ions at $1 \mathrm{GeV} / \mathrm{A}$ (square).

Table 1: Comparison, with several doses, between $\mathrm{SiC}$ and Si detectors

\begin{tabular}{|c|c|c|c|c|c|c|}
\hline & \multicolumn{3}{|c|}{ SiC reverse current $\left[\mathbf{A} / \mathbf{c m}^{3}\right]$} & \multicolumn{3}{c|}{ Si reverse current $\left[\mathbf{A} / \mathbf{c m}^{3}\right]$} \\
\hline Voltage[V] & SiCFresh & $\mathbf{9 4 k G y}$ & $\mathbf{2 1 8 k G y}$ & SiFresh & $\mathbf{9 4 k G y}$ & $\mathbf{2 1 k G y}$ \\
\hline-190 & $1.910^{-6}$ & $8.610^{-6}$ & $4.510^{-4}$ & $8.310^{-5}$ & $3.910^{-4}$ & $8.310^{-5}$ \\
-150 & $8.010^{-7}$ & $5.410^{-6}$ & $2.910^{-4}$ & $6.510^{-6}$ & $3.210^{-4}$ & $8.310^{-5}$ \\
-110 & $3.410^{-7}$ & $3.210^{-6}$ & $1.410^{-4}$ & $4.810^{-6}$ & $2.510^{-4}$ & $8.310^{-5}$ \\
-70 & $1.410^{-7}$ & $1.810^{-6}$ & $3.510^{-5}$ & $2.610^{-6}$ & $7.810^{-5}$ & $3.010^{-5}$ \\
-30 & $7.010^{-8}$ & $6.110^{-7}$ & $3.910^{-7}$ & $7.910^{-7}$ & $6.110^{-5}$ & $2.210^{-5}$ \\
-10 & $4.710^{-8}$ & $2.810^{-7}$ & $5.110^{-8}$ & $1.910^{-7}$ & $3.510^{-5}$ & $1.710^{-5}$ \\
\hline
\end{tabular}

\section{Conclusions}

Silicon Carbide is one of the promising radiation hard materials. Our goal is to realize a two stages detector increasing, beyond the state of the art, thickness and active area. The results of our simulations and test demonstrate that $\mathrm{SiC}$ is a good compromise between several detectors materials. Due to its wide band-gap it has a very low leakage current, good radiation resistance and reasonable sensing capability for charges created during the ionization processes. 


\section{References}

[1]F. Cappuzzello et al. Jour. of Phys. Conf. Ser. 630 (2015) 012018

[2] C. Agodi et al. Nucl. and Part. Phys. Proc. 265266 (2015) 28-30

[3] F. Cappuzzello et al. Eur. Phys. Jour. A,

[4] F. Cappuzzello, C. Agodi, F. Balestra et al. This Conference Proceeding

[5] F. Cappuzzello et al., MAGNEX: an innovative large acceptance spectrometer for nuclear reaction studies, in Magnets: Types, Uses and Safety (Nova Publisher Inc., New York, 2011) pp. 163.

[6] A. Lazzaro, et al.Nucle. Instr. and Meth. A 591 (2008) 394-405

[7]M. Cavallaro. et al. Eur. Phys. J. A (2012) 48- 59.

[8]M.Cavallaro et al. Eur. Phys. Jour. A 48 (2012)59

[9]D. Carbone, F. Cappuzzello and M. Cavallaro Eur. Phys. Jour. A 48 (2012)60

[10]F. Capuzzello. et al. NIM A 638 (2011) 74-82.

[11]A.A. Lebedev et al. Fiz. Tekh. Poluprovodnikov 36. 1354 (2002).

[12]S.M. Sze. Physics of Semiconductor Devices (Ed. Wiley). 1969).

[13]J.F.Ziegler et al. The stopping and range of ions in matter(Ed.

Wiley).(1969). 